\title{
Search for optical flashes of astronomical origin with "Pi of the Sky" prototype
}

\section{A. Majczyna ${ }^{*}{ }^{1}$ M. Należyty, ${ }^{7}$ A. F. Żarnecki, ${ }^{2}$ T. Batsch, ${ }^{1}$ M. Ćwiok, ${ }^{2}$ W. Dominik, ${ }^{2}$ G. Kasprowicz, ${ }^{3}$ A. Majcher, ${ }^{1}$ K. Małek, ${ }^{4}$ L. Mankiewicz, ${ }^{4}$ K. Nawrocki, ${ }^{1}$ R. Pietrzak, ${ }^{5}$ L. W. Piotrowski, ${ }^{2}$ M. Ptasińska, ${ }^{6}$ M. Siudek, ${ }^{6}$ M. Sokołowski, ${ }^{1}$ J. Użycki, ${ }^{1}$ P. Wawer, ${ }^{5}$ R. Wawrzaszek, ${ }^{5}$ G. Wrochna, ${ }^{1}{ }^{M}$. Zaremba, ${ }^{6}$}

${ }^{1}$ The Andrzej Soltan Institute for Nuclear Studies, Hoża 69, 00-681 Warsaw, Poland;

${ }^{2}$ Institute of Experimental Physics, University of Warsaw, Hoża 69, 00-681 Warsaw, Poland;

${ }^{3}$ Institute of Electronic Systems, Warsaw University of Technology, Nowowiejska 15/19, 00-665 Warsaw, Poland;

${ }^{4}$ Center for Theoretical Physics of the Polish Academy of Sciences, Al. Lotników 32/46, 02-668 Warsaw, Poland;

5 Space Research Center of the Polish Academy of Sciences, Bartycka 18A, 00-716 Warsaw, Poland;

${ }^{6}$ Faculty of Physics, Warsaw; University of Technology, Koszykowa 75, 00-662 Warsaw, Poland;

${ }^{7}$ University of Warsaw Astronomical Observatory, Al. Ujazdowskie 4, 00-478 Warsaw, Poland E-mail: agnieszka.majczyna@fuw.edu.pl, nalezyty@astrouw.edu.pl, Filip.Zarnecki@fuw.edu.pl, tadek@ipj.gov.pl Mikolaj.Cwiok@fuw.edu.pl, dominik@fuw.edu.pl, kasprowg@gmail.com majerethetal.cft.edu.pl, malekecft.edu.pl, lech@cft.edu.pl. knawro@gmail.com robertpecbk.waw.pl, Lech-Wiktor.Piotrowski@fuw.edu.pl, maria.ptasinska@gmail.com. gsiudek@cft.edu.pl, Marcin.Sokolowski@fuw.edu.pl. juzycki@fuw.edu.pl, wawerecbk.waw.pl, wawrzaszecbk.waw.pl, wrochna@ipj.gov.pl, xman@if.pw.edu.pl

"Pi of the Sky" is a robotic telescope project aiming for continuous observation of a large part of the sky with high temporal and optical resolution using wide field-of-view instruments. Its primary goal is to look for optical afterglows associated with the gamma ray bursts (GRB), but it is also well suited to study any kind of short time scale astrophysical phenomena. Due to online data analysis in the real time, it has selftriggering capability and can react to external triggers with no time delay. The prototype telescope with two cameras has been installed at Las Campanas Observatory in Chile and is in operation since July 2004. We report on observation of the extraordinarily bright prompt optical emission of GRB 080319B. Combined with the prompt gamma-ray detection and with the following broadband observations of the afterglow, our measurement allowed for a new insight into the nature of GRBs. Other observations of the "Pi of the Sky" prototype, including flare star outbursts and identification of variable stars are also discussed.

European Physical Society Europhysics Conference on High Energy Physics, EPS-HEP 2009,

July 16 - 222009

Krakow, Poland

\footnotetext{
* Speaker.
} 


\section{1. "Pi of the Sky" prototype telescope}

The "Pi of the Sky" telescope consists of two cameras installed on one mount. Each camera is equipped with $2048 \times 2048 \mathrm{CCD}$ sensor and uses Canon telephoto lenses with $\mathrm{f}=85 \mathrm{~cm}$ and $\mathrm{d}=\mathrm{f} / 1.2$. Both detectors observe the same $20^{\circ} \times 20^{\circ}$ field. The telescope located at Las Campanas Observatory can observe stars with R.A. $=\langle 0 \mathrm{~h} ; 24 \mathrm{~h}\rangle$ and Dec $=\left\langle-89.8^{\circ} ;+36^{\circ}\right\rangle$. Up to 2009 we did not use any filter except for IR-cut one in order to maximize the limiting magnitude. The data reduction was made automatically using software and scripts adapted from the ASAS project (Pojmański 1997) or created especially for the "Pi of the Sky". System design and observational strategy determine limiting magnitude to about 10-11 mag for $10 \mathrm{~s}$ exposures and 11-12 mag for $200 \mathrm{~s}$. Our main goal is searching gamma ray bursts (GRB), but also we are interested in different types of variable astronomical objects like: cataclysmic variables symbiotic stars, eclipsing binaries, pulsating stars and other types of variable stars.

\section{Observations by "Pi of the Sky" prototype}

\subsection{GRB080319B}

Early morning March 19th 2008 at 6:12:49 UT "Pi of the Sky" telescope observed very bright object on the sky. Our algorithms identified it as a new, previously unknown object at position R.A. $=14 \mathrm{~h} 31 \mathrm{~m} 42 \mathrm{~s}$, Dec $=+36^{\circ} 18^{\prime} 10^{\prime \prime}$. Few seconds later we obtained GCN alert with information, that Swift satellite instruments observed GRB in this direction.

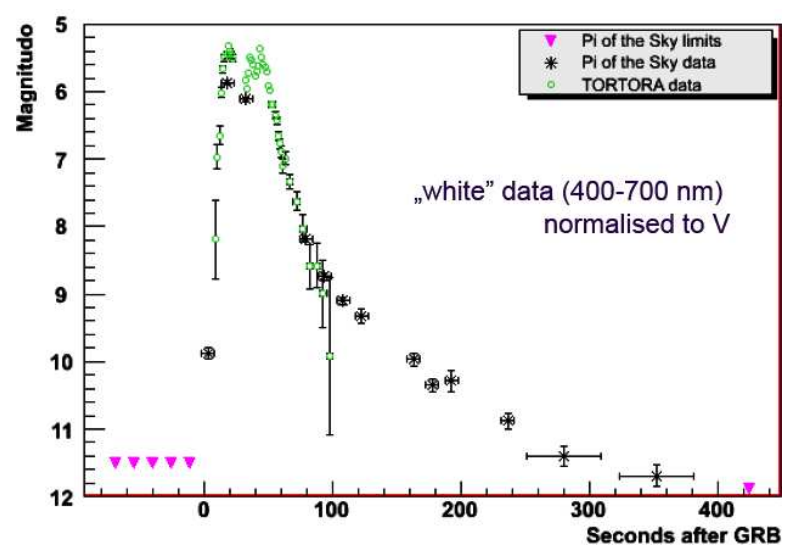

Figure 1: GRB080319B light curve from "Pi of the Sky" and TORTORA data.
Maximum of brightness, measured by $\mathrm{Pi}$ of the Sky was at $5.3 \mathrm{mag}$ and at that moment it could be observed by human naked eye. This is the brightest GRB in optical band ever observed (previous record 9 mag belongs to GRB990123). We made the earliest optical observation during the gamma ray emission. These observations challenged many existing models of GRB emission and demonstrated usefulness of wide-field detectors, capable of continuous monitoring of large parts of the sky. Details about observational properties and theoretical model of this burst was described in Racusin et al. (2008).

\subsection{Flare stars}

Since June 2004 the on-line algorithm detected more than 200 short optical flashes. Two of them were confirmed as flashes of astrophysical origin. The first one was identified as the outburst of CN Leo flare star and the second one as the optical counterpart of GRB080319B. To search for flare stars special off-line algorithm has been also developed. The algorithm takes light curves of stars from the database and looks for signatures of significant brightness increase. As for June 2009 the "Pi of the Sky" the algorithm has identified seven more flare stars (one of flare stars, GJ3334, see Fig. 2). 


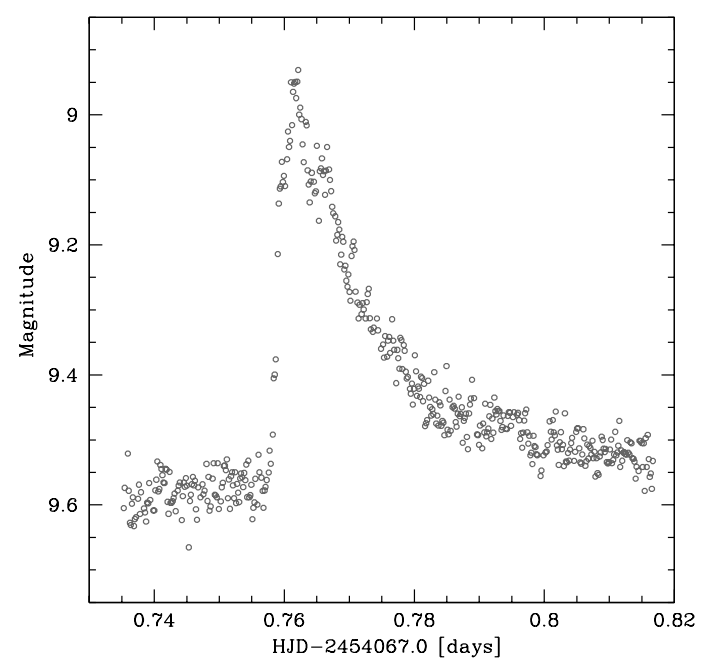

Figure 2: Light curve of the flare star GJ3334.

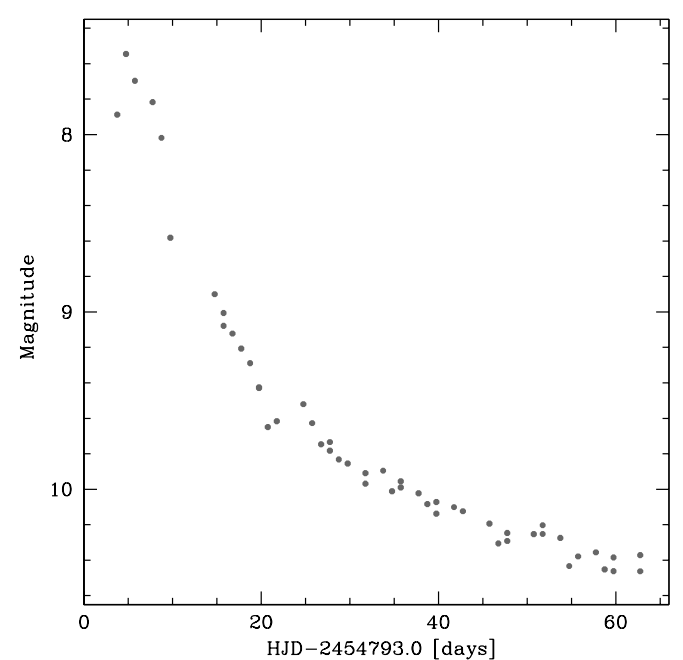

Figure 3: Light curve of the classical nova V679 Car.

\subsection{Classical Novae}

Binary stars consist of a white dwarf and main sequence low mass star. White dwarf accretes matter from the companion via accretion disc. Matter is accumulated on the surface of white dwarf and when the critical mass is reached the thermonuclear flash takes place. In case of classical novae bursts never repeat otherwise object is classified as recurrent nova instead classical (see Warner 1995 for extended review).

Novae identification algorithm uses the data collected during all-sky scans as an input. The algorithm simply looks for a detection of a new object in the data visible on both cameras, on two or more single $10 \mathrm{~s}$ exposures taken one by one and not present in the TYCHO2 database. In November 2008 a classical Nova in Carina (V679 Car, see Fig. 1) was automatically detected by the algorithm. Other most important results were discoveries of two dwarf novae and identification of Nova V5115 Sgr.

\subsection{Periodic variable stars}

"Pi of the Sky" data was checked to search for periodic variable stars. For each such star we find period variability, and determined its type of variability. In this manner catalogue of $\sim 700$ variable stars was prepared based on the observations from 2004-2005 (Majczyna et al. 2008).

\section{References}

[1] A. Majczyna et al. The catalog of short periods stars from the "Pi of the Sky" data, New Astronomy 13 (2008) 414

[2] G. Pojmański The All Sky Automated Survey, Acta Astronomica 47 (1997) 467

[3] J.L. Racusin et al. Broadband observations of the naked-eye $\gamma$-ray burst GRB080319B, Nature 455 (2008) 183

[4] B. Warner Cataclysmic Variable Stars, Cambridge 1995 